\title{
基于嵌入色彩的自适应色彩优化
}

\author{
赵勇威，曾琼”，汪云海，钟凡，屠长河 \\ (山东大学计算机科学与技术学院 青岛 266000) \\ (qiong.zn@sdu.edu.cn)
}

\begin{abstract}
摘 要: 针对基于控制点参数位置的自动色彩优化方法无法有效表达全局数据特征的问题，提出一种基于嵌人色彩 的自适应色彩优化方法，该方法包含色彩嵌人位置估计和嵌人色彩优化 2 个核心过程. 首先基于色彩可辨别性以及 数据分布选取合理的色彩嵌人位置, 然后在固定色彩嵌人位置的基础上, 建立能量优化方程求解最优嵌人色彩, 使 其既满足视觉可辨别, 又能够尽量保证与原始色彩表的一致性. 在此基础上, 开发了一个交互式数据可视探索系统, 该系统可有效支持整体数值感知和局部感兴趣区域探索. 为了评估方法的有效性和系统实用性，在 6 种不同特性的 色彩表、8 种具有不同数据分布的数据集上开展用户实验以及案例分析. 实验表明, 该方法相比其他方法可产生细节 更加丰富的视觉信息, 为用户探索数据提供条件.
\end{abstract}

关键词：数据可视化; 色彩设计; 自动色彩优化

中图法分类号: TP391.41 DOI: 10.3724/SP.J.1089.2021.19266

\section{Adaptive Colormap Optimization Based on Inserting Colors}

\author{
Zhao Yongwei, Zeng Qiong*, Wang Yunhai, Zhong Fan, and Tu Changhe \\ (School of Computer Science and Technology, Shandong University, Qingdao 266000)
}

\begin{abstract}
Traditional automatic color optimization methods face the challenge of expressing global features in dynamic data ranges. To solve this problem, an adaptive colormap optimization method based on inserting colors is proposed, which includes a process of estimating color inserting position and an inserting color optimization procedure. Firstly, color inserting positions are selected based on color discriminability and data histogram distribution. By keeping the color inserting positions, corresponding embedding colors are estimated through a novel energy optimization equation under the guidance of visual discriminability and the consistency to the original colormap. On the basis of the algorithm, an interactive visual data exploratory system is provided, which includes supporting global data perception and local ROI analysis. The effectiveness and applicability of the algorithm is evaluated via a user study and a case study, based on 6 colormaps with different color features and 8 datasets with different data distributions. The results demonstrate that proposed method can produce high quality visual data information compared with other algorithms, providing a condition for further data analysis.
\end{abstract}

Key words: data visualization; color design; automatic color optimization

收稿日期: 2021-07-08; 修回日期: 2021-09-07. 基金项目：国家自然科学基金(61772315, 61861136012). 赵勇威(1996一), 男, 硕 士研究生, 主要研究方向为数据可视化; 曾琼(1987一), 女, 博士, 副研究员, CCF 会员, 论文通讯作者, 主要研究方向为数据可视化; 汪云海(1984-), 男, 博士, 教授, 博士生导师, CCF 会员, 主要研究方向为数据可视化与可视分析; 钟凡(1982一), 男, 博士, 副教 授, 硕士生导师, CCF 会员, 主要研究方向为计算机图形学、增强现实与虚拟现实; 屠长河(1968一), 男, 博士, 教授, 博士生导师, CCF 会员, 主要研究方向为计算机图形学. 
数据可视探索是利用可视化表达从数据中验 证假设、发现规律的过程，该过程中，用户通过观 察、比较、交互等方式获取对数据深层次的认知 ${ }^{[1]}$. 在众多的可视化表达元素中(如形状、纹理、色彩、 透明度、运动等), 色彩是表达标量场数据不可或 缺的可视化编码元素, 应用最为广泛. 色彩可视化 通过建立色彩表与数据之间的映射关系(即色彩映 射), 生成视觉信息丰富的可视化图像.

现有可视化工具(如 ParaView ${ }^{[2]}$ )提供了众多默 认色彩表，如彩虹色彩表和灰度色彩表等. 然而， 传统色彩表中仅包含有限的色彩数量(通常为 256), 导致色彩映射过程中可能丢失原始数据信息. 此 外，人眼无法辨别过于相似的色彩 ${ }^{[3-4]}$, 当色彩表 中色彩差异过低时, 无法观察清楚数据特征. 上述 情况在探索高动态范围数据的局部特征时更为严 重. 因此, 为了获得适合于数据探索任务的色彩 表, 用户需要调节传统色彩表中的色彩位置, 该过 程耗时耗力.

为了提高色彩表设计的效率，研究人员提出 了若干色彩表自动优化方法 ${ }^{[5-7]}$. 如, Tominski 等 ${ }^{[6]}$ 基于直方图均衡化的思想自动调整色彩表中的色 彩位置, 使含有更多数值的数据范围能够有更多 的色彩来表示; Zeng 等 ${ }^{[7]}$ 基于边界模型调整色彩分 布，使数据中隐藏的边界信息得以凸显. 尽管上述 方法能够为特定数值区域(如高密度数值区域 ${ }^{[6]}$ 和 强边界区域 ${ }^{[7]}$ 等)提供更多的视觉信息, 但是由于 原始色彩表中的色彩数目有限, 不可避免会减少 编码其他数值区域的色彩, 难以达到全局平衡, 不 利于数据探索.

针对上述问题，本文提出一种基于嵌人色彩 的自适应色彩优化方法, 其核心思想是在原始色 彩表中特定位置区域引人一种或多种新的色彩(即 嵌人色彩), 以丰富可视化图像中的数据信息. 为 了达到这一目标, 首先, 基于色彩可辨别性以及数 据分布选取合理的色彩嵌人位置; 然后, 基于选定 的色彩嵌人位置提出一种能量优化方程, 以求解 最优嵌人色彩, 使其既满足视觉可辨别, 又能够尽 量保证与原始色彩表的一致性，该方程可利用模 拟退火方法求解. 基于上述色彩优化方法, 开发了 一个交互式数据可视探索系统, 它支持整体数值 感知和(regions-of-interest, ROI)探索等任务, 能够 帮助用户实时地探索数据信息.

为了验证本文方法的有效性，针对 8 种具有不 同数据特征的经典数据集、 6 种具有不同色彩属性 的色彩表(包括感知线性色彩表、两端发散的色彩
表和多色调色彩表), 与同类方法进行了用户比较 实验, 并对本文提出的可视分析系统进行案例分 析. 实验结果表明, 本文方法能够高效地生成丰富 且具有层次感的色彩优化结果, 为用户探索数据 信息提供条件.

\section{1 相关工作}

\section{1 色彩设计准则}

数据可视化领域的色彩设计准则可以分为 2 大类：基于色彩表属性的色彩设计准则及基于数 据和任务的色彩设计准则.

基于色彩表属性的色彩设计准则指的是与色 彩表感知认知属性相关的经验法则, 如色彩的可 辨别性、均匀性和色彩名称等 ${ }^{[8]}$. 上述色彩设计准 则可作为评估色彩表有效性的依据. Bujack 等 ${ }^{[8]}$ 总 结了可视化领域的色彩设计准则, 并基于色彩感 知距离、变化速度等因素, 建立了可辨别性、均匀 性等色彩感知属性的定量数学表达. 此外, 色彩名 称作为一种语言认知因素, 影响着人们对于色彩 的感知. 研究表明, 具有较大色彩名称差异的色彩 表有助于用户感知类别 ${ }^{[9-10]}$, 包含不同色彩名称的 连续色彩表有助于人们观察数据关联关系 ${ }^{[11]}$.

除色彩表属性于色彩设计的影响, 数据可视 化中所涉及的数据特征、分析任务等也对色彩设计 有着重要影响. Bergman 等 ${ }^{[12]}$ 提出针对不同数据类 型、数据频率以及分析任务的色彩设计准则; Reda 等 ${ }^{[13]}$ 设计定性实验，分析了数据空间频率在数值 估计、梯度感知、模式感知等不同任务中对色彩设 计的影响; Dasgupta 等 ${ }^{[14]}$ 通过实验证明了亮度单调 的色彩表对幅值差异感知、空间相似性等任务更为 有效. 因此, 在色彩设计的过程中引入数据特征、 分析任务的影响越来越受到研究人员的关注.

\section{2 自动色彩设计}

色彩设计准则为自动色彩优化提供了理论依 据, 部分研究人员利用亮度一致性、均匀性等色彩 设计准则自动优化色彩表 ${ }^{[15-16]}$. 然而, 此类方法未 考虑数据可视化中数据与任务对于用户感知数据 的影响, 并不适用于可视探索的过程. 在融合数据 与任务的自动色彩优化方面, Tominski 等 ${ }^{[6]}$ 基于数 据直方图分布自动调整色彩表中的控制点色彩位 置, 将更多色彩调整至高密度数据范围区域, 并验 证了该方法在数值感知和数据比较等方面的作用; Zeng 等 ${ }^{[7]}$ 融合边界特征和色彩表保真特征等约束 项构建能量方程, 试图调整色彩表中的色彩位置, 
使更多色彩用于表达含有较为丰富的边界特征数 值区域, 并提供 ROI 的交互方式供用户探索数据 特征. 然而，由于上述优化过程依赖于给定的初始 色彩表, 当调整色彩位置至特定数据范围(如高密 度区域或强边界区域)时，不可避免地会减少编码 其他数据范围中的色彩数目, 难以达到全局平衡, 如图 $1 \mathrm{~b}$ 和图 $1 \mathrm{c}$ 所示, 蓝色箭头区域能看到更多的 层次变化, 但绿色箭头区域变化不够明显.
嵌人色彩是解决上述问题的一个思路，核心 思想是通过在原始色彩表中特定区域嵌人新的色 彩，从而增强原始色彩表在该区域的可辨别性. Samsel 等 ${ }^{[17]}$ 设计了嵌人色彩表集合, 通过嵌人具 有高亮度对比的局部色彩表, 可帮助用户快速认 知数据整体分布. 然而, 目前尚未发现能够自动地 根据数据分布和任务类型选取合适的嵌人色彩的 方法.

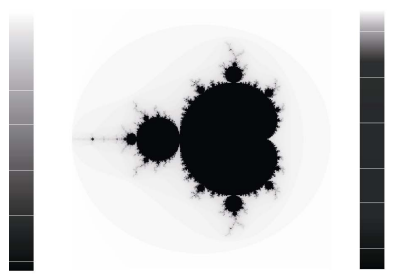

a. 输人色彩表及其可视化

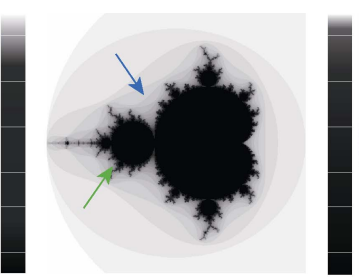

b. Tominski 等 ${ }^{[6]}$

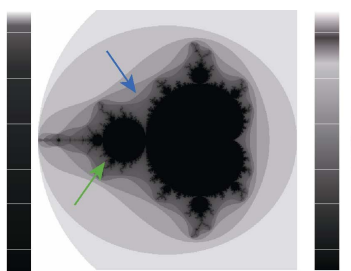

c. Zeng 等 ${ }^{[7]}$

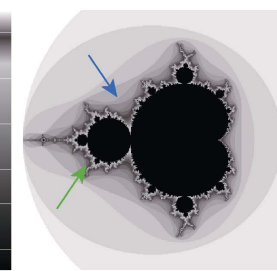

d. 本文

图 13 种方法在 Mandelbrot 分形数据上的可视化结果

\section{3 交互式色彩设计工具}

尽管自动色彩优化方法为用户探索数据提供 条件, 但是基于动态交互的数据可视探索过程仍 然是用户理解和发掘数据规律的重要途径. Elmqvist 等 ${ }^{[1]}$ 基于“概率+细节”模式构建了交互可视探 索系统 Color Lens, 它可根据用户 ROI 调整初始色 彩表，也可提供新的色彩表用于编码多个局部 ROI. 与基于控制点调整的自动色彩优化方法 ${ }^{[6-7]}$ 类 似，其缺陷在于凸显局部 ROI 时会减弱其他数值区 域的数值, 不利于比较 ROI 与其他区域的数值; 同 样, 利用多个色彩表编码不同 ROI 也存在数据与色 彩一一映射关系丢失的问题，即同一数值可能由多 种不同的色彩表示. 为了让用户能够观察到高动态 范围数据中的局部细节信息, Samsel 等 ${ }^{[4]}$ 提出了基 于色彩嵌人的色彩表设计交互系统 ColorMoves, 其 通过在特定区域嵌人新的色彩表，使用户能够观 察 ROI 更为细微的数据特征, 然而用户需要手动 选取嵌人色彩表及其嵌人位置.

\section{2 基于嵌入色彩的色彩设计}

根据文献调研以及非正式专家访谈，本文总 结了 3 点色彩优化设计过程中应遵循的设计需求.

DR1. 最大化不同数据分布下的视觉可辨别 性, 尽可能呈现丰富的数据信息, 使用户能够快速 观察数据的全局变化 ${ }^{[5-7]}$.

DR2. 尽可能保持默认色彩表的分布，以减少 色彩表变化过大而导致的用户感知突变 ${ }^{[7,14,18]}$.
DR3. 提供便捷的交互, 帮助用户探索数据中 局部 ROI 的信息, 支持多个局部区域的数据比较 与分析 ${ }^{[1,7]}$.

尽管现有色彩优化方法强调设计需求 DR1, 但其本质在于依据数据特征自动调整色彩表中各 控制点的位置, 这一优化过程以损耗其他数据特 征区域的可辨别性为代价, 难以达到全局平衡. 为 了解决这一问题，本文提出基于嵌人色彩的色彩 优化, 试图通过在特定区域嵌人色彩补偿提高视 觉可辨别性，该过程不影响其他数据特征区域的 色彩感知. 此外, 由于嵌人色彩可能导致原始色彩 表发生较大变化, 因此, 该过程需要尽可能地满足 设计需求 DR2, 即嵌人色彩应尽可能地减少对原 始色彩表的侵人. 综上，如何估计色彩嵌人的位置 以及如何选择合理的嵌人色彩是本文方法需要解 决的 2 个核心问题.

定义 1. 给定包含 $l$ 个数据样本的标量场数据 $D$ 和初始色彩表 $\boldsymbol{C}$, 该色彩表包含 $n$ 种 CIELab 空 间的三维色彩，这些色彩可由 $O(o<n)$ 个控制点通 过线性插值而生成. 具体而言, 控制点包含位置 $p=\left\{p_{1}, p_{2}, \cdots, p_{o}\right\}$ 和颜色 $\boldsymbol{c}=\left\{\boldsymbol{c}_{1}, \boldsymbol{c}_{2}, \cdots, \boldsymbol{c}_{o}\right\} 2$ 种属性， 任意 2 个相邻控制点 $i$ 和 $j$ 之间的颜色集合表示为 $c_{i j}=\left\{c_{i j}^{1}, c_{i j}^{2}, \cdots, c_{i j}^{k}\right\}$. 通过将数据和色彩标准化至同 一数值区间(如 $0 \sim 1$ ), 可基于索引建立数据与色彩 之间的关联映射, 从而获得色彩编码的可视化图 像. 本文的目标在于寻找合理的色彩嵌人位置 $\bar{p}$ 和嵌人色彩 $\overline{\boldsymbol{c}}$, 使色彩编码的可视化图像中能够 
表现出层次丰富的数据信息.

\section{1 色彩嵌入位置估计}

色彩编码可视化图像存在视觉可辨别性低的 原因在于数据与色彩的分布不一致, 特别是数据 特征区域所对应的色彩可辨别性较低，即对应较 少的编码色彩, 视觉上会损失更多的数据信息. 因 此, 本文提出了一个度量数据特征与色彩分布不 一致性的算子，即

$$
I_{i j}=\mathrm{e}^{\left(w_{i j}-d_{i j}\right)}
$$

该度量子描述了色彩表中相邻控制点 $i$ 和 $j$ 之 间数据特征与色彩分布之间的矛盾一当数据特 征越多的区域所对应色彩可辨别性较低时，不一 致性度量子数值较大, 即数据特征与色彩分布的 不一致性越强; 反之则数值越小, 两者之间的矛盾 越小. 式(1)中 $w_{i j}$ 描述了控制点 $i$ 和 $j$ 之间的数据 特征, 采用数据分布密度计算, 即 $w_{i j}=\frac{l_{i j}}{l}$. 其中, $l_{i j}$ 为控制点 $i$ 和 $j$ 之间对应数据样本的总和; $I$ 为 整个二维标量场数据样本的总和. 此外, 式(1)中的 $d_{i j}$ 为相邻控制点 $i$ 和 $j$ 之间的色彩可辨别性 ${ }^{[8]}$, 即

$$
d_{i j}=\frac{1}{k-1} \sum_{s \neq r}^{k} \frac{\Delta E\left(\boldsymbol{c}_{s}, \boldsymbol{c}_{r}\right)}{|s-r|}
$$

其中, $\Delta E$ 为 CIELab 空间欧几里得度量 ${ }^{[19]} ; k$ 为 控制点 $i$ 和 $j$ 之间的色彩数目, $s$ 和 $r$ 为控制点 $i$ 和 $j$ 之间的某个色彩位置.

为了满足 DR1, 本文将在高密度和低色彩可 辨别性区域嵌人新的色彩, 即选取数据与色彩分 布不一致性最大的 $m$ 个数值区域作为色彩嵌人区 间, 并以其各自中点作为色彩嵌人位置 $\bar{p}=\left\{\bar{p}_{1}, \bar{p}_{2}, \cdots, \bar{p}_{m}\right\}$. 默认情况下，本文设 $m=1$, 用 户可调节数值区域数目, 以生成更为丰富的可视 化结果.

\section{2 嵌入色彩优化}

根据 DR1 和 DR2, 嵌人色彩既要满足色彩可 辨别性需求, 也要避免对原始色彩表造成过度修 改. 因此, 本文提出利用根据色彩名称选择嵌人 色彩，其优势在于，同一色彩名称既对应多种不 同属性的色彩，所对应色彩集合又具有较高的和 谐性 ${ }^{[9,11,20]}$, 不会引起用户的感知突变. 因此, 本 文基于上述思想建立能量方程, 以优化求解合理 的嵌人色彩，即

$$
\underset{\overline{\boldsymbol{c}}}{\arg \min } E(\overline{\boldsymbol{c}} \mid i, j)=\alpha * D(\overline{\boldsymbol{c}} \mid i, j)+N(\overline{\boldsymbol{c}} \mid i, j)
$$

其中, $i$ 和 $j$ 均为色彩嵌人位置所对应的控制点; $D(\overline{\boldsymbol{c}} \mid i, j)$ 为色彩可辨别性约束项; $N(\overline{\boldsymbol{c}} \mid i, j)$ 为色 彩名称约束项; $\alpha$ 为两者之间的平衡参数, 其作 用是平衡色彩可辨别性约束与色彩名称约束之间 的矛盾, 第 2.3 节将进一步探讨该参数对于嵌人色 彩结果的影响.

色彩可辨别性约束项旨在计算基于嵌人色彩 所生成色彩表的可辨别性. 本文通过线性插值将 嵌人色彩 $\bar{c}$ 融人控制点 $i$ 和 $j$ 之间，从而为该控制 点区间生成新色彩数值. 由于式(3)中目标值为最 小优化，因此，色彩可辨别性约束项定义为

$$
D(\overline{\boldsymbol{c}} \mid i, j)=\frac{1}{d_{i j}(\overline{\boldsymbol{c}})}
$$

其中, $\boldsymbol{d}_{i j}(\overline{\boldsymbol{c}})$ 为在新色彩表上控制点 $i$ 和 $j$ 之间所 求解的可辨别性, 计算过程与式(2)一致. 该方程 可使优化过程选择可辨别性高的色彩.

色彩名称约束项旨在计算嵌人色彩与其周围 色彩的差异性，为了简化计算，本文仅考虑嵌人色 彩与其邻域控制点之间的差异，即

$$
N(\overline{\boldsymbol{c}} \mid i, j)=g\left(\overline{\boldsymbol{c}}, \boldsymbol{c}_{i}\right) * g\left(\overline{\boldsymbol{c}}, \boldsymbol{c}_{j}\right)
$$

其中, $g\left(\overline{\boldsymbol{c}}, \boldsymbol{c}_{i}\right)$ 和 $g\left(\overline{\boldsymbol{c}}, \boldsymbol{c}_{j}\right)$ 分别为嵌人色彩与嵌人区 间控制点之间的色彩名称差异，该方程倾向于选 择与相邻控制点更接近的色彩作为优化解. 根据 文献[19]中的方法, 色彩名称差异可通过色彩名称 矩阵间的最小余弦距离求解，即

$$
g\left(\overline{\boldsymbol{c}}, \boldsymbol{c}_{i}\right)=1-\cos \left(\boldsymbol{T}_{\overline{\boldsymbol{c}}}-\boldsymbol{T}_{\boldsymbol{c}_{i}}\right)=1-\frac{\boldsymbol{T}_{\overline{\boldsymbol{c}}} \boldsymbol{T}_{\boldsymbol{c}_{i}}}{\left\|\boldsymbol{T}_{\bar{c}}\right\| \boldsymbol{T}_{\boldsymbol{c}_{i}} \|}
$$

其中, $\boldsymbol{T}_{\boldsymbol{c}_{i}}$ 为色彩 $\boldsymbol{c}_{i}$ 所对应的名称矩阵 ${ }^{[9]}$.

本文利用模拟退火方法 ${ }^{[21]}$ 求解式(3), 该方法 计算过程简单、通用且鲁棒性强, 可用于求解复杂 的非线性优化问题. 具体实现中, 本文将初始温度 设为 $100{ }^{\circ} \mathrm{C}$, 温度下降系数设为 0.85 , 共迭代 300 次. 经过多次测试, 在相同的参数下, 尽管模拟退 火方法可能产生不同结果, 但所产生的色彩具有 较高的相似性.

\section{3 参数分析}

本文方法涉及色彩嵌入区间数目 $m$ 和色彩优 化平衡参数 $\alpha$ 这 2 个可调参数, 本节将阐述不同 参数设置对结果的影响.

$m$ 用于控制嵌人色彩区间的个数，使数据与 色彩的分布不一致性最大的 $m$ 个区间被选为色彩 嵌人区间. 图 2 展示了不同嵌人色彩数目所产生的 结果, 由图 $2 \mathrm{~b}$ 图 $2 \mathrm{~d}$ 分别选取数据特征与色彩分 
布不一致性最大的前 1 3 个嵌人区间, 并在 $\alpha=10$ 的设置下进行嵌人色彩选取. 随着 $m$ 的增加，可 视化图像中呈现的整体数据变化信息更为丰富.

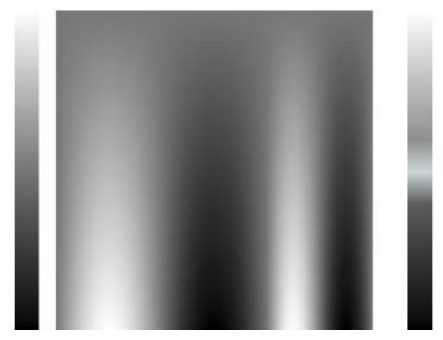

a. 输入数据及其色彩表

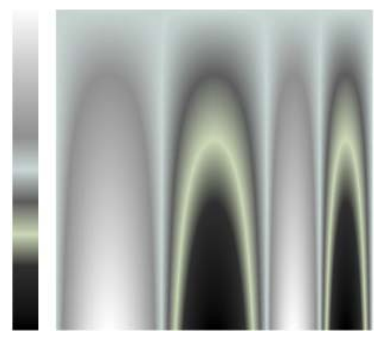

c. $m=2$

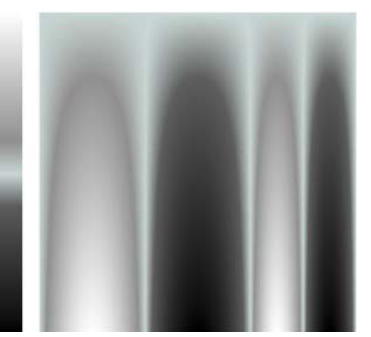

b. $m=1$

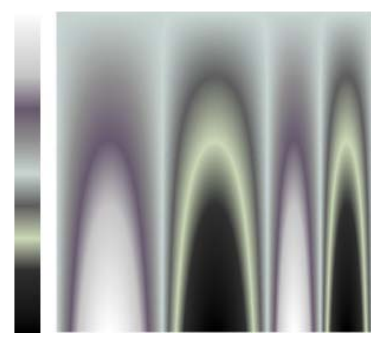

d. $m=3$
图 2 不同的 $m$ 取值产生的嵌人结果

$\alpha$ 用于控制嵌人色彩能量方程中不同约束项 的作用. $\alpha$ 越大, 则色彩可辨别性约束项作用越 强, 所选取的嵌人色彩可能与原始色彩表具有较 大出人; $\alpha$ 越小, 选取的嵌人色彩越接近于原始 色彩表中的颜色. 图 3 呈现了不同 $\alpha$ 取值对结果 的影响，随着 $\alpha$ 增大，优化方法所选取的嵌人色 彩与输人色彩表差异越大, 使嵌人色彩区域具有 更强的可辨别性，从而呈现更多细节信息。

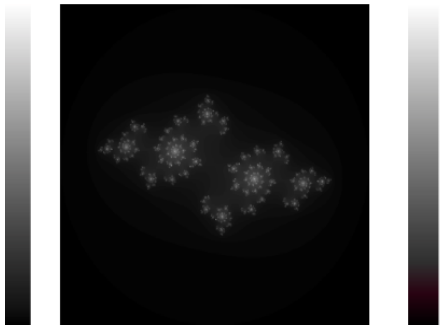

a. 输人灰度色彩表及可视化

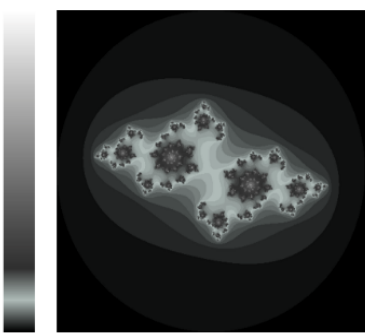

c. $\alpha=35$

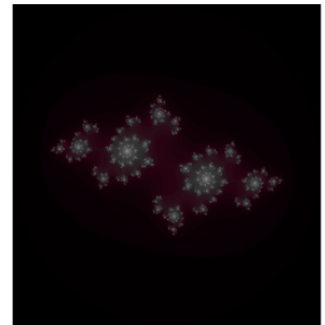

b. $\alpha=25$

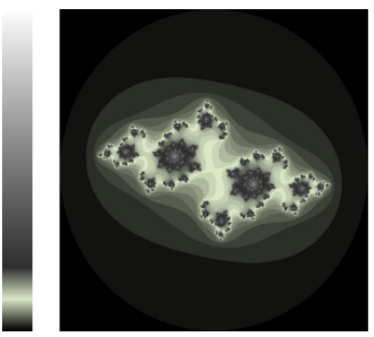

d. $\alpha=45$
图 3 不同的 $\alpha$ 取值产生的嵌人结果

\section{3 基于色彩嵌入的可视探索系统}

基于上述核心方法, 本文开发了一个基于色 彩嵌人的数据可视探索系统. 该系统包括色彩表 视图、输人视图、输出视图、数据特征视图、色彩 表可辨别性视图以及历史视图等模块, 系统界面 如图 4 所示.

（1）色彩表视图. 该视图中包含 70 种内置色 彩表, 覆盖常用的 50 种感知线性色彩表、9 种两端 分散的色彩表和 11 种多色调非线性色彩表, 用户 可选择任意色彩表作为初始色彩编码.

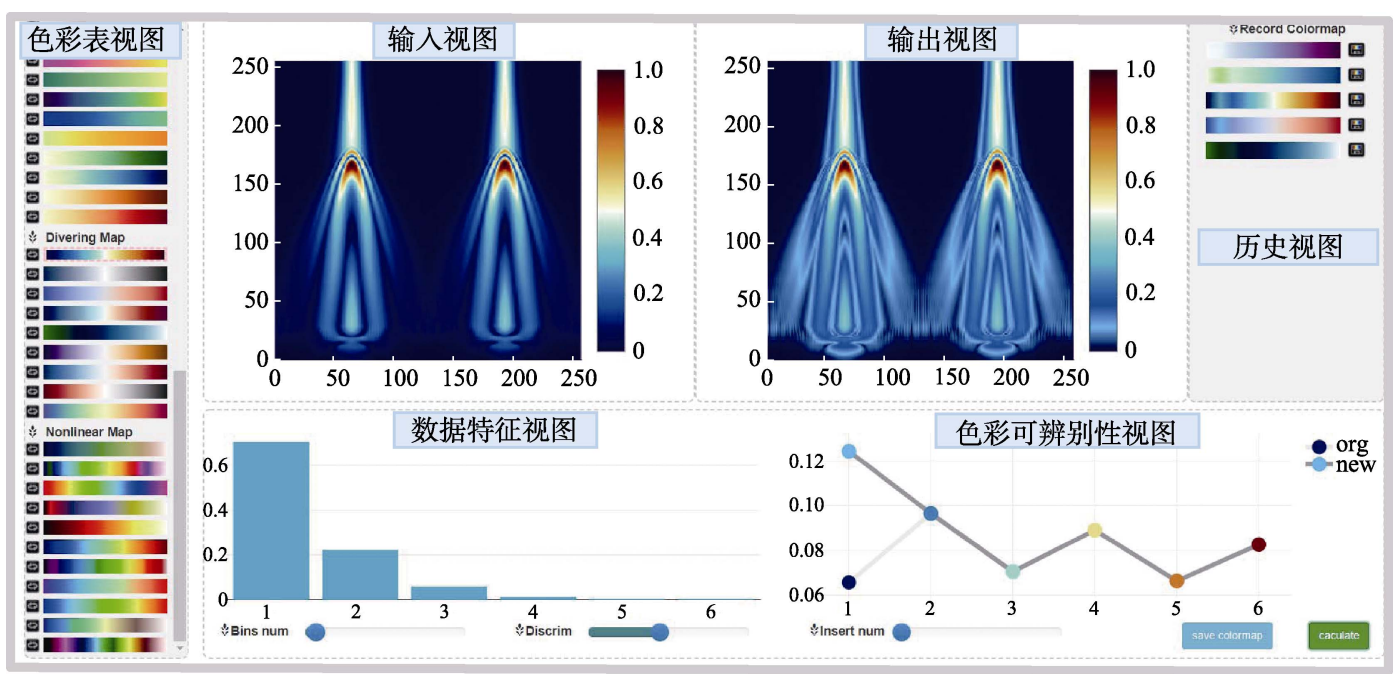

图 4 基于嵌人色彩的可视系统界面

(2) 输人视图. 该视图用于呈现初始数据的色 彩编码可视化结果并支持用户交互. 用户可通过
拖动导人原始数据, 也可通过滑动鼠标滚轮缩放 可视化图像. 此外, 用户可通过鼠标左键在可视化 
图像上选择单个或多个 ROI, 系统将根据用户所 选择区域中的数据分布优化初始色彩表, 使用户 能够着重观察比较 ROI 内部细节.

(3) 输出视图. 该视图用于呈现色彩优化后的 可视化图像, 同样支持数据缩放和 ROI 选择. 需要 说明的是，在输出视图上的 ROI 将以该视图上的 色彩表为优化对象，而非初始输人色彩表.

(4) 数据特征视图. 该视图用于展现各相邻控 制点之间的数据直方图分布，数据越多，则相应的 柱状图越高，用户可拖动 Bins 滑动条调节控制点 的数目.

（5）色彩可辨别性视图. 该视图用于呈现初始 色彩表与优化后的色彩表在各控制点之间所对应 的色彩可辨别性变化曲线. 浅灰色折线段为原始 色彩表的可辨别性曲线，深灰色折线段则对应新 生成的色彩表. 可以看出，在第 1 个控制点区间嵌 人色彩之后，该区间的色彩可辨别性相比原始色 彩表明显提升. 在用户交互探索的过程中，该视图 中色彩可辨别性曲线会发生联动变化

(6) 历史视图. 该视图用于展示用户探索过程 中产生的色彩表, 用户可回溯探索过的色彩表, 并 快速保存满意的结果.

\section{4 实验与结果分析}

本文方法基于 Matlab 实现，采用 JavaScript 实 现交互式数据探索系统, 实验结果在配置为 Intel Core i7-6700 K (4 GHz GPU), 16 GB 内存的台式机 上完成. 本文与 2 种同类方法开展了用户对比实验,
以评估方法的有效性，并邀请领域专家使用交互式 系统并进行案例分析，以验证该系统的实用性.

\section{1 用户实验}

为了证明本文方法在色彩优化方面的有效 性，采用直方图均衡化方法 ${ }^{[6]}$ 和基于边界色彩优 化方法 ${ }^{[7]}$ 开展用户实验，并对所生成的实验结果 进行分析.

（1）色彩表. 本文采用 6种常见色彩表(包含感 知线性的色彩表、两端分散的色彩表和多色调非线 性色彩表各 2 种)开展实验. 图 5a 所示为实验中所 涉及的色彩表及其 CIELab 空间各分量分布. 其中 黑色曲线表示亮度分量; 红色表示绿-红分量; 蓝色 表示蓝-黄分量. 可以看出, 本文实验采用了具有不 同分布特性的色彩表，以避免评估的片面性.

(2) 数据集. 本文基于 8 种具有不同数据分布 特性的常见数据集(含 3 种合成数据和 5 种真实数 据)开展实验. D1 和 $\mathrm{D} 3$ 为不同尺度的 Mandelbrot 分形数据, D2 为频率变化函数合成的数据 ${ }^{[22],} \mathrm{D} 4$ 为欧洲海水盐度数据, D5 和 D6 为不同视角无人机 电磁辐射数据 ${ }^{[23]}, \mathrm{D} 7$ 和 D8 分别为肺部正电子发射 型计算机断层显像 (positron emission computed tomography, PET)数据和脑部放射治疗剂量数据. 图 $5 \mathrm{~b}$ 所示为各数据在灰度色彩表编码下的可视化 图像，每幅色彩表的下面都呈现了该数据中各数 据值(水平方向)与其一阶导(垂直方向)之间的对应 关系散点图, 散点的颜色表示在某一数据分布下 的样本点数量, 颜色越深, 则数目越多. 如图 $5 \mathrm{~b}$ 所 示, 本文所采用的实验数据并不偏向于特定数据 分布.
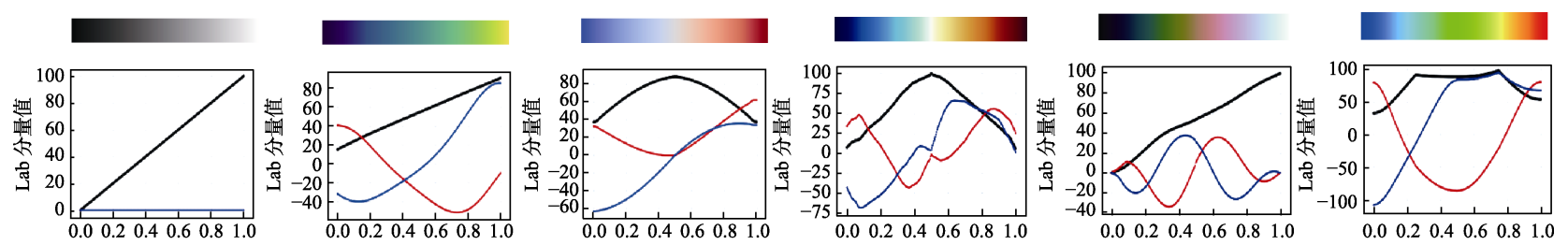

a. 实验色彩表及其 CIELab 空间各分量分布
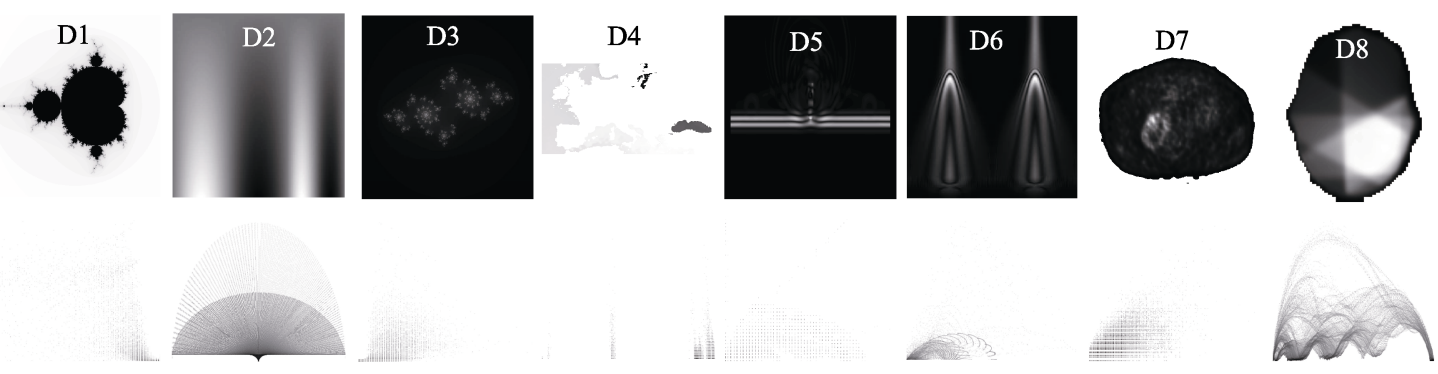

b. 实验数据和各数据值(水平方向)及其一阶导(垂直方向)对应关系散点图

图 5 本文用户实验中所采用的色彩表和数据 
（3）实验数据生成过程. 基于以上数据集和颜 色图，通过分别采用本文方法、Tominski 等 ${ }^{[6]}$ 方法、 Zeng 等 ${ }^{[7]}$ 方法分别生成 48 种优化后的可视化结果, 实验中共包含 $48 \times 3=144$ 种实验结果. 这 3 种方法 均选择 5 个初始控制点, 本文实验结果都在参数 $m=1, \alpha=40$ 下生成, Zeng 等 ${ }^{[7]}$ 方法以及 Tominski 等 ${ }^{[6]}$ 方法的结果均在统一最优参数下生成.

（4）实验任务. 实验过程中，每位用户被要求 从 2 个数据可视化中选择“具有更为丰富数据信 息”的可视化表达，这 2 个实验数据包含一个本文 实验结果和一个同类方法(Tominski 等 ${ }^{[6]}$ 方法或 Zeng 等 ${ }^{[7]}$ 方法) 所生成的结果, 每位用户需要做 96 组上述实验.

（5）实验用户. 本文邀请 20 位用户参与实验, 其中包含 10 位女性和 10 位男性，年龄为 22 28 岁. 本文先基于 14 幅 Ishihara 色盘对用户进行色盲测 试，当且仅当准确率高于 $85 \%$ (最多错 2 个)时才能 够参加实验. 本文实验在固定室内光照环境下开 展, 显示器分辨率为 $1920 \times 1080$. 实验结束后, 每位用户均得到 $¥ 30$ 的回报.

(6) 实验过程. 该实验包含 3 部分：训练过程、 主实验过程和非正式访谈. 其中，主实验过程中用 户首先比较 48 组本文方法与某一种同类方法进行 实验，休息约 $12 \mathrm{~h}$ ，继续比较 48 组本文方法与另一 种同类方法. 为了减少学习效应, 实验过程中随机 打散了不同数据的出现顺序，以及每一数据中不同 方法的排列位置. 在实验过程中, 用户点击鼠标并 选择具有更丰富数据信息的可视化表达，实验系统 将自动记录用户的选择和实验时间. 总体而言, 每
个用户在主实验过程平均耗时为 $15 \mathrm{~min}$ (不考虑休 息时间), 其中最少耗时 $7 \mathrm{~min}$, 最长耗时 $23 \mathrm{~min}$.

(7) 实验结果. 实验共收集了 1920 组实验数 据, 分别包含 960 组本文与 Tominski 等 ${ }^{[6]}$ 方法的比 较(简记为“本文/Tominski 等 ${ }^{[6]}$ ”), 以及本文与 Zeng

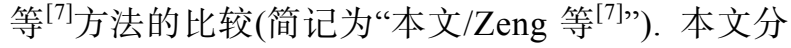
析并对比了同类方法，用户选择本文方法“展现更 多细节”的比值(简记为“平均百分比对比”), 该数 值越高, 表明更多的用户认为本文方法比同类方 法能够呈现出更多细节信息, 反之, 则用户认可数 较少.

图 6a 所示为实验结果统计分析图, 相比同类 方法，更多的用户认为本文方法所生成的可视化 结果能够表达更为丰富的数据信息, 这一优势在 与 Tominski 等 ${ }^{[6]}$ 方法相比时更为明显. 图 $6 \mathrm{~b}$ 所示 为在不同色彩表上对实验数据进行统计分析的结 果, 可以看出, 在所有色彩表上本文方法均优于同 类方法, 表明本文方法的性能受色彩表分布的影 响不大. 图 $6 \mathrm{c}$ 所示为在不同数据上、不同方法实 验数据的统计分析结果, 尽管本文方法在所有数 据上均优于 Tominski 等 ${ }^{[6]}$ 方法, 但是在 D2, D4 和 D8 的表现上低于 Zeng 等 ${ }^{[7]}$ 方法. 究其原因, 这 3 组数据在多个数据区域具有明显变化, 而在实验 过程中为了保证公平性, 本文固定在一个嵌人区 间求解结果，因此无法有效表达其他数值区域的 变化信息. 本文方法的表现与数据分布具有一定 关联关系, 其本质在于估计嵌人位置时需要考虑 数据分布, 因此, 当数据中存在较多具有变化信息 的区域时, 需要进一步调节色彩嵌人区域的数量.

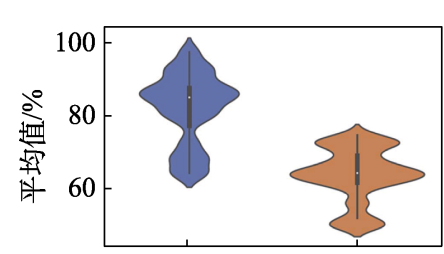

a. 所有实验数据平均对比

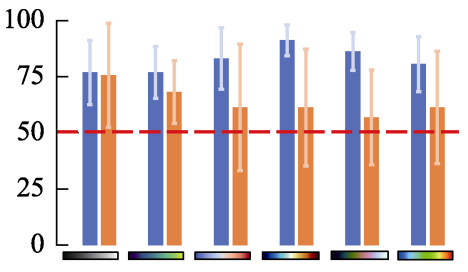

b. 不同颜色图上的平均对比

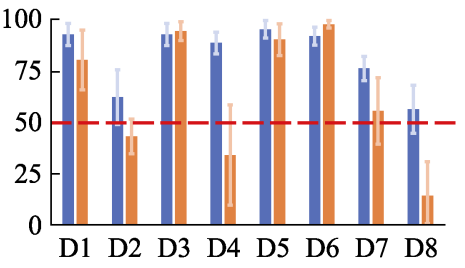

c. 不同数据上的平均对比

$\square$ 本文/Tominski 等[6];

本文/Zeng等 ${ }^{[7]}$

图 6 用户实验统计结果

\section{2 案例分析}

邀请一位数据分析专家利用本文的交互式系 统探索红海附近的海气热通量数据变化情况. 该 专家导人一个利用 WRF-ARW 模型生成的红海附 近海气热通量数据，其数据数值范围为 -264 $144 \mathrm{~W} / \mathrm{s}^{2}$, 在非洲沿岸的南部具有较大值而北部数
值相对较低. 由于最大值与最小值具有较大差异, 原始输人色彩表不能同时表现低值部分内部和高 值部分内部的海气热通量变化. 该专家首先使用 ROI 工具选出感兴趣的亚丁湾区域的位置, 如图 $7 \mathrm{a}$ 所示. 系统自动嵌人浅橙色, 呈现出更丰富的 热通量变化, 如图 $7 \mathrm{~b}$ 所示. 更进一步, 专家缩小亚 
丁湾海域的范围, 并增加了埃及东部的海域, 初始 交互如图 7c 所示, 系统可自动根据相应区域的数 据嵌人多种色彩. 如图 $7 \mathrm{~d}$ 所示, 优化后的色彩分 布使专家能够同时观察海气热通量低值区域与高 值区域的数据变化信息. 总的来说, 专家认为本文 系统能够便捷地展示更为丰富的数据变化信息, 有助于探索不同数值区域的数据变化, 对系统的 实时交互表示满意, 但认为嵌人色彩附近的精确 数值读取略为困难.

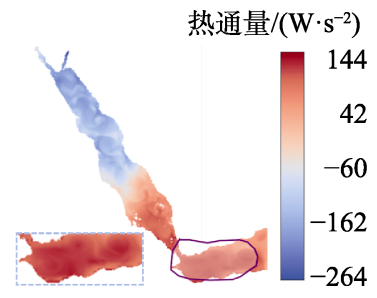

a. 输人和单 $\mathrm{ROI}$

热通量 $/\left(\mathrm{W} \cdot \mathrm{s}^{-2}\right)$

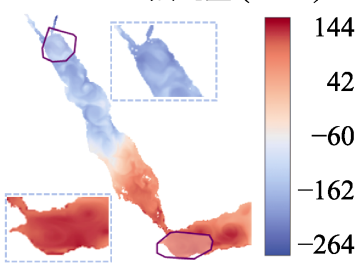

c. 输人和多 ROI

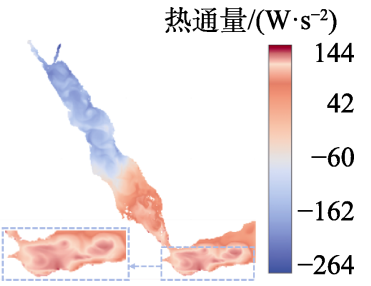

b. 基于单 ROI 的优化结果

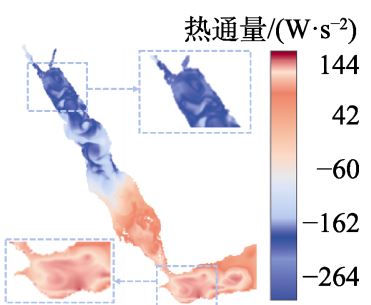

d. 基于多 ROI 的优化结果
图 7 案例分析交互结果示意

\section{5 结 语}

本文提出了一种基于嵌人色彩的自适应色彩 优化方法, 其基于数据特征估计色彩嵌人位置, 利用色彩可辨别性和色彩名称等约束建立嵌人色 彩优化方程. 在此基础上, 本文开发了一个交互 式数据可视探索系统, 该系统可有效地支持整体 数值感知以及局部 ROI 分析等可视探索过程. 本 文针对 6 种具有不同色彩特征的色彩表、8 种不 同数据分布特征的数据集, 开展用户实验评估本 文方法的有效性，并邀请领域专家使用本系统并 评估其实用性. 本文以二维静态标量场数据阐述 并评估方法, 该方法可以直接拓展至二维时序标 量场数据.

虽然本文方法可直接拓展于三维数据，但是 由于三维数据可视化中需要考虑透明度、光照、视 角等因素, 其色彩优化问题更为复杂, 仅采用本文 方法未必能得到最为合理的嵌人色彩. 如何在三 维数据进行色彩优化是未来可探索的方向. 此外, 由于本文方法侧重于展现前景数据变化信息, 在
求解嵌人色彩过程中没有考虑色彩顺序和背景色 彩的影响. 因此, 生成的色彩表可能存在色彩感知 无序、与背景色彩区分度过低等问题. 在将该方法 应用于精确的数值读取任务时, 需进一步引人色 彩有序性和背景色彩等约束.

\section{参考文献(References):}

[1] Elmqvist N, Dragicevic P, Fekete J. Color Lens: adaptive color scale optimization for visual exploration[J]. IEEE Transactions on Visualization and Computer Graphics, 2011, 17(6): 795-807

[2] Ahrens J, Geveci B, Law C. ParaView: an end-user tool for large data visualization[M] //Visualization Handbook. Amsterdam: Elsevier, 2005

[3] Ware C, Turton T L, Bujack R, et al. Measuring and modeling the feature detection threshold functions of colormaps[J]. IEEE Transactions on Visualization and Computer Graphics, 2019, 25(9): 2777-2790

[4] Samsel F, Klaassen S, Rogers D. ColorMoves: real-time interactive colormap construction for scientific visualization[J]. IEEE Computer Graphics and Applications, 2018, 38(1): 20-29

[5] Schulze-Wollgast P, Tominski C, Schumann H. Enhancing visual exploration by appropriate color coding[C] //Proceedings of International Conference in Central Europe on Computer Graphics, Visualization and Computer Vision. Plzen: University of West Bhenmia, 2005: 203-210

[6] Tominski C, Fuchs G, Schumann H. Task-driven color coding[C] //Proceedings of the 12th International Conference Information Visualisation. Los Alamitos: IEEE Computer Society Press, 2008: 373-380

[7] Zeng Q, Wang Y Q, Zhang J, et al. Data-driven colormap optimization for 2D scalar field visualization[C]//Proceedings of the IEEE Visualization Conference. Los Alamitos: IEEE Computer Society Press, 2019: 267-271

[8] Bujack R, Turton T L, Samsel F, et al. The Good, the bad, and the ugly: a theoretical framework for the assessment of continuous colormaps[J]. IEEE Transactions on Visualization and Computer Graphics, 2018, 24(1): 923-933

[9] Heer J, Stone M. Color naming models for color selection, image editing and palette design[C] //Proceedings of the SIGCHI Conference on Human Factors in Computing Systems. New York: ACM Press, 2012: 1007-1016

[10] Chuang J, Stone M, Hanrahan P. A probabilistic model of the categorical association between colors[C]//Proceedings of Color Imaging Conference. Virginia: Society for Imaging Science and Technology, 2008: 6-11

[11] Reda K, Szafir D A. Rainbow revisited: modeling effective colormap design for graphical inference[J]. IEEE Transactions on Visualization and Computer Graphics, 2021, 27(2): 10321042 
[12] Bergman L D, Rogowitz B E, Treinish L A. A rule-based tool for assisting colormap selection[C] //Proceedings of the 6 th Conference on Visualization. Los Alamitos: IEEE Computer Society Press, 1995: 118-125

[13] Reda K, Nalawade P, Ansah-Koi K. Graphical perception of continuous quantitative maps: The effects of spatial frequency and colormap design[C] //Proceedings of the SIGCHI Conference on Human Factors in Computing Systems. New York: ACM Press, 2018: 1-12

[14] Dasgupta A, Poco J, Rogowitz B, et al. The effect of color scales on climate scientists' objective and subjective performance in spatial data analysis tasks[J]. IEEE Transactions on Visualization and Computer Graphics, 2020, 26(3): 1577-1591

[15] Sisneros R, Raji M, van Moer M W, et al. Chasing rainbows: a color theoretic framework for improving and preserving bad colormaps[C] //Proceedings of International Symposium on Visual Computing. Heidelberg: Springer, 2016: 391-402

[16] Nuñez J R, Anderton C R, Renslow R S. Optimizing colormaps with consideration for color vision deficiency to enable accurate interpretation of scientific data[J]. PLoS One, 2018, 13(7): e0199239

[17] Samsel F, Overmyer T, Navratil P A. Highlight insert color- maps: luminance for focused data analysis[C] //Proceedings of Eurographics Conference on Visualization. Aire-la-Ville: Eurographics Association Press, 2019: 55-59

[18] Zhou L, Hansen C D. A survey of colormaps in visualization[J]. IEEE Transactions on Visualization and Computer Graphics, 2016, 22(8): 2051-2069

[19] Sharma G, Wu W C, Dalal E N. The CIEDE2000 color-difference formula: implementation notes, supplementary test data, and mathematical observations[J]. Color Research \& Application, 2005, 30(1): 21-30

[20] Lu K C, Feng M, Chen X, et al. Palettailor: discriminable colorization for categorical data[J]. IEEE Transactions on Visualization and Computer Graphics, 2021, 27(2): 475-484

[21] Aarts E, Korst J. Simulated annealing and Boltzmann machines: a stochastic approach to combinatorial optimization and neural computing[M]. New York: John Wiley \& Sons, 1989

[22] Nardini P, Chen M, Bujack R, et al. A testing environment for continuous colormaps[J]. IEEE Transactions on Visualization and Computer Graphics, 2021, 27(2): 1043-1053

[23] Cao Y, Mo Z Y, Ai Z W, et al. Parallel visualization of large-scale multifield scientific data[J]. Journal of Visualization, 2019, 22: 1107-1123 